Research Article

\title{
Best Proximity Point for the Sum of Two Non-Self-Operators
}

\author{
V. Pragadeeswarar, ${ }^{1}$ R. Gopi, ${ }^{1}$ and M. De la Sen $\mathbb{D}^{2}$ \\ ${ }^{1}$ Department of Mathematics, Amrita School of Engineering, Amrita Vishwa Vidyapeetham, Coimbatore, India \\ ${ }^{2}$ Institute of Research and Development of Processes IIDP, University of the Basque Country, Campus of Leioa, P.O. Box 48940, \\ Leioa, Bizkaia, Spain
}

Correspondence should be addressed to M. De la Sen; manuel.delasen@ehu.eus

Received 13 April 2020; Accepted 22 May 2020; Published 8 June 2020

Academic Editor: Tepper L Gill

Copyright (c) 2020 V. Pragadeeswarar et al. This is an open access article distributed under the Creative Commons Attribution License, which permits unrestricted use, distribution, and reproduction in any medium, provided the original work is properly cited.

\begin{abstract}
In the present paper, we focus our attention on the existence of the fixed point for the sum of the cyclic contraction and the noncyclic accretive operator. Also, we study the best proximity point for the sum of two non-self-mappings. Moreover, we provide the existence of the best proximity point for the cyclic contraction through the notion of the nonlinear $\mathscr{D}$-set contraction. Finally, we give the existence of the best proximity point for the sum of the nonlinear $\mathscr{D}$-set contraction mapping and partially completely continuous mapping in the setting of the partially ordered complete normed linear space.
\end{abstract}

\section{Introduction}

Fixed point theory plays an important role in the area of nonlinear functional analysis, and it has many applications in the study of nonlinear differential and integral equations. The study of nonlinear equations of the form $\Gamma_{1} \eta+\Gamma_{2} \eta=\eta$, where $\Gamma_{1}, \Gamma_{2}: \mathscr{B} \longrightarrow \mathscr{B}$ are mappings on the Banach space $\mathscr{B}$, helps to solve many physical nonlinear real-life problems. For example, Dhage and Otrocol [1] gave the existence and approximation of solutions to the following hybrid differential equation:

$$
\left\{\begin{array}{l}
x^{\prime}(t)=f(t, x(t))+g\left(t, \max _{a \leq \xi \leq t} x(\xi)\right), \\
x(a)=\alpha_{0} \in \mathbb{R}
\end{array}\right.
$$

for all $t \in J=[a, b]$ and $f, g: J \times \mathbb{R} \longrightarrow \mathbb{R}$ are continuous functions. Also, Banaś and Amar [2] obtained the existence of the solution to the nonlinear integral equation of the form

$$
x(t)=a(t)+\int_{0}^{1} k(t, s) f(s, x(s)) \mathrm{d} s+\int_{0}^{1} u(t, s, x(s)) \mathrm{d} s,
$$

for $\quad t \in J=[a, b]$ and $a \in L^{1}(J), f: J \times \mathbb{R} \longrightarrow \mathbb{R}$, $k: J \times J \longrightarrow \mathbb{R}^{+}$, and $u: J \times J \times \mathbb{R} \longrightarrow \mathbb{R}$. So, the researchers involved in finding the solution of the equation $\Gamma_{1} \eta+\Gamma_{2} \eta=\eta$, which is clearly the problem of finding the sufficient condition for the existence of fixed point for the sum of two mappings.

In the sequel, in 1955, Krasnoselskii gave an existence of the solution for the equation $\Gamma_{1} \eta+\Gamma_{2} \eta=\eta$ in the Banach space setting, where $\Gamma_{1}$ is the contraction and $\Gamma_{2}$ is the compact operator. Later, many researchers extended Krasnoselskii's theorem in different directions (see [3-7] and the references therein). Vijayaraju [7] proved the theorems pointing the existence of the fixed point for a sum of nonexpansive and continuous mappings and also a sum of asymptotically nonexpansive and continuous mappings in the setting of locally convex spaces. O'Regan [5] established the fixed point theorem for the sum of two operators $\Gamma_{1}+\Gamma_{2}$ if $\Gamma_{1}$ is compact and $\Gamma_{2}$ is nonexpansive. Moreover, the results were used to prove the existence of the solution for second-order boundary value problem. O'Regan and Taoudi [6] proved the fixed point theorems for the sum of two weakly sequentially continuous mappings in the Banach space. Dhage [3] proved the fixed point result by combining two fixed point theorems of Krasnoselskii and Dhage and also derived the existence result for the product of two operators in Banach algebra. Dhage [4] found the local 
version of fixed point theorems of Krasnoselskii and Nashed et al. By using this result, he provided the application to nonlinear functional integral equations.

Agarwal et al. [8] obtained the existence of the fixed point for two mappings, compact mapping and nonexpansive mapping, in the setting of both the weak and the strong topology of a Banach space. Ben Amar and GarciaFalset [9] proved the existence of fixed point theorems for different kinds of contractions such as nonlinear weakly condensing, 1-set weakly contractive, and pseudo-contractive and nonexpansive operators defined on unbounded domains and provided application to generalized Hammerstein integral equations. Arunchai and Plubtieng [10] improved the Krasnoselskii theorem on fixed points for the sum of operators $\Gamma_{1}+\Gamma_{2}$, where $\Gamma_{1}$ is the weakly-strongly continuous mapping and $\Gamma_{2}$ is the asymptotically nonexpansive mapping. Banaś and Amar [2] proved some new types of fixed point theorems for the sum of $\Gamma_{1}+\Gamma_{2}$ on an unbounded closed convex subset of a Hausdorff topological vector space, which are used to provide the solution of integral equations in the Lebesgue space. Wang [11] derived fixed point results for the sum of two operators $\Gamma_{1}$ and $\Gamma_{2}$ if $\Gamma_{1}$ is contractive with respect to the measure of weak noncompactness and $\Gamma_{2}$ is the $\phi$-nonlinear contraction in the setting of the Banach space. By having this result, he obtained the existence of solutions to a nonlinear Hammerstein integral equation in the $L^{1}$ space. Ben Amar et al. [12] improved Krasnoselskii-type fixed point results for the equation $\left(\Gamma_{1}+\Gamma_{2}\right) x=x$, where the operator $\Gamma_{1}$ is $(w s)$-compact and $\Gamma_{2}$ is (ws)-compact and asymptotically $\Phi$-nonexpansive operator on an unbounded closed convex subset of a Banach space.

Later, the people were interested in finding the fixed point in partially ordered metric spaces which are more general than metric spaces. First, Ran and Reurings [13] initiated the fixed point theorems for the contraction mappings in the partially ordered metric space, which is further improved by Nieto and Rodríguez-López [14] and by Petruşel and Rus [15], and used to give the existence of the solution for boundary value problems of nonlinear firstorder ordinary differential equations.

Recently, the researchers show interest in finding the optimum solution for real-time modeling problems. In this direction, instead of studying about the fixed point (whenever the fixed point does not exist), the researchers are working on approximating the fixed point in some sense, known as the best proximity point. The existence of best proximity point theorems helps to obtain the optimum solution for different types of modelings. In the literature, there are more number of articles about the existence of the best proximity point for single operators. For example, Basha [16] derived best proximity point theorems for proximal contractions of the first and second kind in the setting of the metric space. Basha [17] proved the existence of the best proximity point for principal cyclic contractive mappings, proximal cyclic contractive mappings, and proximal contractive mappings which are defined on the metric space. In 2000, Eldred and Veeramani [18] proved the best proximity point result for contractive type mappings in the metric space. Al-Thagafi and Shahzad [19] obtained convergence and existence results of the best proximity points for cyclic $\phi$-contraction maps in the metric space. For more existence of the best proximity point results, we refer the reader to [20-25].

In the light of the above literature survey, we want to find the approximate solution for the fixed point equation of the form $\left(\Gamma_{1}+\Gamma_{2}\right) x=x$, where $\Gamma_{1}, \Gamma_{2}$ are non-self-mappings. So, in this work, we initiate to study the best proximity point for the sum of two non-self-operators, and we provide the existence of the best proximity point for the sum of two operators using best proximity point theorems for the single operator. Additionally, we prove an existence result of the fixed point for the sum of cyclic and noncyclic operators, which involves the concept of accretive operators. Finally, we discuss some notions of the ordered normed linear space, and we find sufficient conditions for the existence of the best proximity point in this space.

\section{Preliminaries}

First, we collect some notions from [26]. Throughout the paper, we denote $\mathscr{B}, \mathscr{X}$ as the Banach space and metric space, respectively, and let

$$
\mathscr{B}^{*}=\{f: \mathscr{B} \longrightarrow \mathbb{R} \mid f \text { is continuous linear transformation }\}
$$

be its dual. For each $\eta \in \mathscr{B}$, we associate the set

$$
J(\eta)=\left\{f \in \mathscr{B}^{*}:\langle\eta, f\rangle=\|\eta\|^{2}=\|f\|^{2}\right\},
$$

where $\langle\eta, f\rangle$ denotes $f(\eta)$. The multivalued operator $J: \mathscr{B} \longrightarrow \mathscr{B}^{*}$ is called the duality mapping of $\mathscr{B}$. Suppose $\Gamma$ is an operator from $\mathscr{B}$ to $\mathscr{B}$. Then, the operator $-\Gamma: \mathscr{B} \longrightarrow \mathscr{B}$ is defined by $-\Gamma(\eta)=-\Gamma \eta$.

Here, we give the definition for weak accretive via the accretive operator in [26].

Definition 1. An operator $\Gamma: \mathscr{B} \longrightarrow \mathscr{B}$ is said to be weak accretive if for $\left(\eta_{1}, \Gamma \eta_{1}\right),\left(\eta_{2}, \Gamma \eta_{2}\right) \in \mathscr{B} \times \mathscr{B}$, there exists $\varphi \in J\left(\eta_{1}-\eta_{2}\right)$ such that $\left\langle\Gamma \eta_{1}-\Gamma \eta_{2}, \varphi\right\rangle \geq 0$, where $J$ is as in (4).

The following lemma is helpful for our one of the results.

Lemma 1. If $\Gamma$ is weak accretive on $\mathscr{B}$, then $\left\|\eta_{1}-\eta_{2}\right\| \leq\left\|\eta_{1}-\eta_{2}+\lambda\left(\Gamma \eta_{1}-\Gamma \eta_{2}\right)\right\|$, for all $\lambda \geq 0$, and $\eta_{1}, \eta_{2} \in \mathscr{B}$.

Proof. Let $\eta_{1}, \eta_{2} \in \mathscr{B}$ with $\eta_{1} \neq \eta_{2}$. Since $\Gamma$ is weak accretive, for $\left(\eta_{1}, \Gamma \eta_{1}\right),\left(\eta_{2}, \Gamma \eta_{2}\right) \in \mathscr{B} \times \mathscr{B}$, there exists $\varphi \in J\left(\eta_{1}-\eta_{2}\right)$ such that $\left\langle\Gamma \eta_{1}-\Gamma \eta_{2}, \varphi\right\rangle \geq 0$. Now, we have

$$
\begin{aligned}
\left\|\eta_{1}-\eta_{2}\right\|^{2} & =\left\langle\eta_{1}-\eta_{2}, \varphi\right\rangle \\
& \leq\left\langle\eta_{1}-\eta_{2}, \varphi\right\rangle+\lambda\left\langle\Gamma \eta_{1}-\Gamma \eta_{2}, \varphi\right\rangle \\
& =\left\langle\eta_{1}-\eta_{2}+\lambda\left(\Gamma \eta_{1}-\Gamma \eta_{2}\right), \varphi\right\rangle \\
& \leq\left\|\eta_{1}-\eta_{2}+\lambda\left(\Gamma \eta_{1}-\Gamma \eta_{2}\right)\right\|\|\varphi\| \\
& =\left\|\eta_{1}-\eta_{2}+\lambda\left(\Gamma \eta_{1}-\Gamma \eta_{2}\right)\right\|\left\|\eta_{1}-\eta_{2}\right\| .
\end{aligned}
$$


Theorem 1 (see [27]). Let $\mathscr{M}, \mathcal{N}$ be nonempty subsets of $\mathscr{X}$ which are complete. Suppose $\Gamma: \mathscr{M} \cup \mathcal{N} \longrightarrow \mathscr{M} \cup \mathcal{N}$ satisfies

(1) $\Gamma(\mathscr{M}) \subseteq \mathscr{N}$ and $\Gamma(\mathscr{N}) \subseteq \mathscr{M}$.

(2) $d(\Gamma \eta, \Gamma \xi) \leq k d(\eta, \xi)$ for $\eta \in \mathscr{M}, \xi \in \mathcal{N}$,

where $0<k<1$. Then, there exists $\tau \in \mathscr{M} \cap \mathcal{N}$ such that $\Gamma(\tau)=\tau$.

\section{Main Results}

Theorem 2. Let $\mathscr{M}, \mathcal{N}$ be subsets of $\mathscr{B}$ such that $\mathscr{M} \cup \mathcal{N}$ is the subspace. Let $\Gamma_{1}, \Gamma_{2}: \mathscr{M} \cup \mathcal{N} \longrightarrow \mathscr{M} \cup \mathcal{N}$, where $\Gamma_{1}$ and $\Gamma_{2}$ are cyclic and noncyclic mappings which satisfy the following:

(1) $-\Gamma_{2}$ is the weak accretive operator, and $\Gamma_{1}$ is onto

(2) $\left(I-\Gamma_{2}\right)$ is invertible on $\Gamma_{1}(\mathscr{M} \cup \mathcal{N})$, where $I$ is the identity operator

(3) $\eta=\Gamma_{2} \eta+\Gamma_{1} \xi, \xi \in \mathscr{M} \Longrightarrow \eta \in \mathscr{N}$ $\eta=\Gamma_{2} \eta+\Gamma_{1} \xi, \xi \in \mathcal{N} \Longrightarrow \eta \in \mathscr{M}$

and

(4) $\left\|\Gamma_{1} \eta-\Gamma_{1} \xi\right\| \leq k\|\eta-\xi\|, k \in(0,1)$, for $\eta \in \mathscr{M}$ and $\xi \in \mathscr{N}$

Then, there exists $\tau \in \mathscr{M} \cup \mathcal{N}$ such that $\left(\Gamma_{1}+\Gamma_{2}\right) \tau=\tau$.

Proof. First, we note that assumption (3) is valid only if $\mathscr{M} \cup \mathcal{N}$ is the subspace. Since $\Gamma_{1}$ is onto, $\Gamma_{1}(\mathscr{M} \cup \mathcal{N})=\mathscr{M} \cup \mathcal{N}$. Since $\left(I-\Gamma_{2}\right)$ is invertible on $\Gamma_{1}(\mathscr{M} \cup \mathscr{N})$, we can define

$$
\left(I-\Gamma_{2}\right)^{-1} \Gamma_{1}: \mathscr{M} \cup \mathcal{N} \longrightarrow \mathscr{M} \cup \mathcal{N}
$$

Now, we show that $\left(I-\Gamma_{2}\right)^{-1} \Gamma_{1}$ is the cyclic mapping. Let $\xi \in \mathscr{M}$; then, $\Gamma_{1} \xi \in \mathscr{N}$. Since $\left(I-\Gamma_{2}\right)$ is invertible on $\Gamma_{1}(\mathscr{M} \cup \mathscr{N})$, there exists unique $\eta$ such that $\left(I-\Gamma_{2}\right)^{-1} \Gamma_{1} \xi=\eta$. Then, the equation $\eta=\Gamma_{2} \eta+\Gamma_{1} \xi$ has a unique solution $\eta$. Then, by (3), we obtain $\left(I-\Gamma_{2}\right)^{-1} \Gamma_{1} \xi \in \mathcal{N}$. Then, $\left(I-\Gamma_{2}\right)$ ${ }^{-1} \Gamma_{1}(\mathscr{M}) \subseteq \mathcal{N}$. Similarly, we can prove $\left(I-\Gamma_{2}\right)^{-1} \Gamma_{1}(\mathscr{N}) \subseteq \mathscr{M}$. Next, we prove that $\left(I-\Gamma_{2}\right)^{-1}$ is nonexpansive on $\Gamma_{1}(\mathscr{M} \cup \mathcal{N})$. Since $\left(I-\Gamma_{2}\right)$ is invertible on $\Gamma_{1}(\mathscr{M} \cup \mathcal{N})$, it implies that $R(I-$ $\left.\Gamma_{2}\right)=\Gamma_{1}(\mathscr{M} \cup \mathcal{N})$, where $R\left(I-\Gamma_{2}\right)$ is the range of $I-\Gamma_{2}$. Let $\eta, \xi \in \Gamma_{1}(\mathscr{M} \cup \mathcal{N})$; then, there exist $z_{1}, z_{2} \in \Gamma_{1}(\mathscr{M} \cup \mathcal{N})$ such that $\left(I-\Gamma_{2}\right)^{-1} \eta=z_{1},\left(I-\Gamma_{2}\right)^{-1} \xi=z_{2}$. Using Lemma 1, we get

$$
\begin{aligned}
\|\eta-\xi\| & =\left\|z_{1}-\Gamma_{2} z_{1}-\left(z_{2}-\Gamma_{2} z_{2}\right)\right\| \\
& =\left\|z_{1}+\left(-\Gamma_{2} z_{1}\right)-\left(z_{2}+\left(-\Gamma_{2} z_{2}\right)\right)\right\| \\
& =\left\|z_{1}-z_{2}+\left(\left(-\Gamma_{2} z_{1}\right)-\left(-\Gamma_{2} z_{2}\right)\right)\right\| \\
& \geq\left\|z_{1}-z_{2}\right\| \\
& =\left\|\left(I-\Gamma_{2}\right)^{-1} \eta-\left(I-\Gamma_{2}\right)^{-1} \xi\right\| .
\end{aligned}
$$

Therefore, for $\eta \in \mathscr{M}, \xi \in \mathcal{N}$, we obtain

$$
\begin{aligned}
\left\|\left(I-\Gamma_{2}\right)^{-1} \Gamma_{1} \eta-\left(I-\Gamma_{2}\right)^{-1} \Gamma_{1} \xi\right\| & \leq\left\|\Gamma_{1} \eta-\Gamma_{1} \xi\right\| \\
& \leq k\|\eta-\xi\| .
\end{aligned}
$$

Then, the operator $\left(I-\Gamma_{2}\right)^{-1} \Gamma_{1}$ agrees with the hypothesis of Theorem 1 , and then there is $\tau \in \mathscr{M} \cap \mathcal{N}$ such that $\left(I-\Gamma_{2}\right)^{-1} \Gamma_{1} \tau=\tau$, which implies $\left(\Gamma_{2}+\Gamma_{1}\right) \tau=\tau$.

\section{Best Proximity Point Theorems}

The following notions are used in this article: let $\mathscr{M}, \mathscr{N}$ be nonempty subsets of $\mathscr{X}$.

$$
\begin{aligned}
d(\mathscr{M}, \mathscr{N}) & =\inf \{d(\eta, \xi): \eta \in \mathscr{M}, \xi \in \mathscr{N}\}, \\
\mathscr{M}_{0} & =\{\eta \in \mathscr{M}: d(\eta, \xi \prime)=d(\mathscr{M}, \mathcal{N}) \text { for some } \xi \prime \in \mathcal{N}\}, \\
\mathcal{N}_{0} & =\{\xi \in \mathscr{N}: d(\eta \prime, \xi)=d(\mathscr{M}, \mathcal{N}) \text { for some } \eta \prime \in \mathscr{M}\} .
\end{aligned}
$$

The pair $(\mathscr{M}, \mathscr{N})$ is said to have $P$-property if for $\eta_{1}, \eta_{2} \in \mathscr{M}$ and $\xi_{1}, \xi_{2} \in \mathcal{N}$,

$$
\left\{\begin{array}{l}
d\left(\eta_{1}, \xi_{1}\right)=d(\mathscr{M}, \mathcal{N}), \\
d\left(\eta_{2}, \xi_{2}\right)=d(\mathscr{M}, \mathcal{N}),
\end{array} \Longrightarrow d\left(\eta_{1}, \eta_{2}\right)=d\left(\xi_{1}, \xi_{2}\right) .\right.
$$

Definition 2. A function $\Gamma: \mathscr{X} \longrightarrow \mathscr{X}$ is called Lipschitzian if there exists a real constant $\kappa \geq 0$ such that, for all $\eta, \xi \in \mathscr{X}$,

$$
d(\Gamma(\eta), \Gamma(\xi)) \leq \kappa d(\eta, \xi)
$$

Definition 3 (see [20]). Let $\mathscr{M}, \mathcal{N}$ be nonempty subsets of $\mathscr{X}$. A map $\Gamma: \mathscr{M} \longrightarrow \mathscr{N}$ is said to be a weakly contractive mapping if

$$
d(\Gamma \eta, \Gamma \xi) \leq d(\eta, \xi)-\chi(d(\eta, \xi))
$$

for all $\eta, \xi \in \mathscr{M}$, where $\chi:[0, \infty) \longrightarrow[0, \infty)$ is a nondecreasing and continuous function such that $\chi$ is positive on $(0, \infty), \chi(0)=0$ and $\lim _{t \rightarrow \infty} \chi(t)=\infty$.

Theorem 3 (see [20]). Let $\mathscr{X}$ be complete and $\mathscr{M}, \mathcal{N} \subseteq \mathscr{X}$ be nonempty, closed sets such that $\mathscr{M}_{0}$ is nonempty. Let $\Gamma: \mathscr{M} \longrightarrow \mathcal{N}$ be a weakly contractive mapping such that $\Gamma\left(\mathscr{M}_{0}\right) \subseteq \mathcal{N}_{0}$. Assume the pair $(\mathscr{M}, \mathcal{N})$ has the P-property. Then, there exists unique $\eta^{*}$ in $\mathscr{M}$ such that $d\left(\eta^{*}, \Gamma \eta^{*}\right)=d(\mathscr{M}, \mathcal{N})$.

The following theorem tells that the sum of two non-selfoperators has the best proximity point in Banach space settings. The notion $(1 / 2) \mathcal{N}=\{\eta: \eta=(b / 2), b \in \mathcal{N}\}$, where $\mathcal{N}$ is the subset of $\mathscr{B}$, is used in the following theorem.

Theorem 4. Let $\mathscr{M}, \mathcal{N}$ be two nonempty convex, closed subsets of $\mathscr{B}$. Assume $\mathscr{M}_{0}$ is nonempty. Let $\Gamma_{1}, \Gamma_{2}: \mathscr{M} \longrightarrow \mathscr{N}$ be two mappings which satisfy

(1) If $\eta \in \mathscr{M}$, then $\Gamma_{1} \eta+\Gamma_{2} \eta \in \mathcal{N}$

(2) $\Gamma_{1}$ is $(k / 2)$-Lipschitzian, where $k \in(0,1]$

(3) $\left\|\Gamma_{2} \eta-\Gamma_{2} \xi\right\| \leq(k / 2)\|\eta-\xi\|-\chi(\|\eta-\xi\|)$, for all $\eta, \xi \in \mathscr{M}$, where $\chi$ is as in Definition 3 and $k \in(0,1]$

(4) $\Gamma_{1}\left(\mathscr{M}_{0}\right) \subseteq(1 / 2) \mathcal{N}_{0}$ and $\Gamma_{2}\left(\mathscr{M}_{0}\right) \subseteq(1 / 2) \mathcal{N}_{0}$

If the pair $(\mathscr{M}, \mathcal{N})$ has the P-property, then there exists unique $\eta^{*}$ in $\mathscr{M}$ such that $\left\|\eta^{*}-\left(\Gamma_{1}+\Gamma_{2}\right) \eta^{*}\right\|=d(\mathscr{M}, \mathcal{N})$. 
Proof. Because of (1), we define $\Gamma_{1}+\Gamma_{2}: \mathscr{M} \longrightarrow \mathcal{N}$ by $\left(\Gamma_{1}+\Gamma_{2}\right) \eta=\Gamma_{1} \eta+\Gamma_{2} \eta$. First, we show that $\Gamma_{1}+\Gamma_{2}$ satisfies $\left(\Gamma_{1}+\Gamma_{2}\right)\left(\mathscr{M}_{0}\right) \subseteq \mathscr{N}_{0}$. For $\eta \in \mathscr{M}_{0}$, we have $\Gamma_{1}(\eta) \in(1 / 2) \mathcal{N}_{0}$ and $\Gamma_{2}(\eta) \in(1 / 2) \mathscr{N}_{0}$. Therefore, there exist $\xi_{1}, \xi_{2} \in \mathcal{N}_{0}$ such that $\Gamma_{1} \eta=\left(\xi_{1} / 2\right), \Gamma_{2} \eta=\left(\xi_{2} / 2\right)$. Then, $\left(\Gamma_{1}+\Gamma_{2}\right)(\eta)=\Gamma_{1} \eta+\Gamma_{2} \eta=\left(\xi_{1} / 2\right)+\left(\xi_{2} / 2\right)$. Since $\mathcal{N}_{0}$ is convex, $\quad\left(\Gamma_{1}+\Gamma_{2}\right)(\eta) \in \mathscr{N}_{0}$. This shows $\left(\Gamma_{1}+\Gamma_{2}\right)\left(\mathscr{M}_{0}\right) \subseteq \mathcal{N}_{0}$. Now we prove the mapping $\Gamma_{1}+\Gamma_{2}$ is weakly contractive. For $\eta, \xi \in \mathscr{M}_{0}$, we have

$$
\begin{aligned}
\left\|\left(\Gamma_{1}+\Gamma_{2}\right)(\eta)-\left(\Gamma_{1}+\Gamma_{2}\right)(\xi)\right\| & =\left\|\Gamma_{1} \eta-\Gamma_{1} \xi+\Gamma_{2} \eta-\Gamma_{2} \xi\right\| \\
& \leq\left\|\Gamma_{1} \eta-\Gamma_{1} \xi\right\|+\left\|\Gamma_{2} \eta-\Gamma_{2} \xi\right\| \\
& \leq \frac{k\|\eta-\xi\|}{2}+\frac{k\|\eta-\xi\|}{2}-\chi(\|\eta-\xi\|) \\
& =k\|\eta-\xi\|-\chi(\|\eta-\xi\|) \\
& \leq\|\eta-\xi\|-\chi(\|\eta-\xi\|) .
\end{aligned}
$$

This gives $\Gamma_{1}+\Gamma_{2}$ is a weakly contractive mapping from $\mathscr{M}_{0}$ to $\mathscr{N}_{0}$. Clearly, the pair $\left(\mathscr{M}_{0}, \mathscr{N}_{0}\right)$ has the $P$-property, and $d\left(\mathscr{M}_{0}, \mathcal{N}_{0}\right)=d(\mathscr{M}, \mathcal{N})$. Then, the map $\Gamma_{1}+\Gamma_{2}$ satisfies the requirements of Theorem 3 , and there exists $\eta^{*} \in \mathscr{M}_{0}$ such that $\left\|\eta^{*}-\left(\Gamma_{1}+\Gamma_{2}\right)\left(\eta^{*}\right)\right\|=d(\mathscr{M}, \mathcal{N})$.

\section{Best Proximity Point Theorems in the Ordered Normed Linear Space}

In this section, first we extract some notions from [28] to obtain best proximity point results. Let $\mathscr{V}$ be the real vector space. The pair $(\mathscr{V}, \prec)$ is called the partially ordered linear space, where $\prec$ is the partial order. Two elements $\eta, \xi \in \mathscr{V}$ are called comparable if either $\eta \prec \xi$ or $\xi \prec \eta$ holds. A nonempty subset $\mathscr{C}$ of $\mathscr{V}$ is said to be a chain or totally ordered if any two elements of $\mathscr{C}$ are comparable. The space $(\mathscr{V}, \prec,\|\cdot\|)$ is called the partially ordered normed linear space, where $\|\cdot\|$ is the norm on $\mathscr{V}$.

Definition 4 (see [28]). A mapping $\Gamma: \mathscr{V} \longrightarrow \mathscr{V}$ is called monotone nondecreasing if $\eta \prec \xi$ implies $\Gamma \eta \prec \Gamma \xi$ for all $\eta, \xi \in \mathscr{V}$. Similarly, $\Gamma$ is called monotone nonincreasing if $\eta \prec \xi$ implies $\Gamma \xi \prec \Gamma \eta$ for all $\eta, \xi \in \mathscr{V}$.

Definition 5 (see [28]). A subset $\mathscr{C}$ of $(\mathscr{V}, \prec,\|\cdot\|)$ is called partially bounded if every chain in $\mathscr{C}$ is boünded.

We denote by $P_{b d, c h}(\mathscr{V}), P_{r c p, c h}(\mathscr{V})$ the family of all bounded chains and relatively compact chains of $\mathscr{V}$, respectively.

Definition 6 (see [28]). A mapping $\mu^{p:} P_{b d, c h}(\mathscr{V}) \longrightarrow \mathbb{R}^{+}=$ $[0, \infty)$ is called a partial measure of noncompactness in $\mathscr{V}$ if it satisfies

(1) $\varnothing \neq\left(\mu^{p}\right)^{-1}(0) \subset P_{r c p, c h}(\mathscr{V})$.

(2) $\mu^{p}(\bar{D})=\mu^{p}(D)$.

(3) $\mu^{p}$ is nondecreasing, i.e., if $D_{1} \subset D_{2} \Longrightarrow$ $\mu^{p}\left(D_{1}\right) \leq \mu^{p}\left(D_{2}\right)$.
(4) If sequence $\left\{D_{n}\right\}$ of closed chains in $P_{b d, c h}(\mathscr{V})$ with $D_{n+1} \subset D_{n}(n=1,2, \ldots)$ and if $\lim _{n \longrightarrow \infty} \mu^{p}\left(D_{n}\right)=0$, then the set $\bar{D}_{\infty}=\cap_{n=1}^{\infty} D_{n}$ is nonempty.

The notion in (1) is known as the kernel of $\mu^{p}$, that is,

$$
\operatorname{ker} \mu^{p}=\left\{D \in P_{b d, c h}(\mathscr{V}): \mu^{p}(D)=0\right\}
$$

Clearly, $\operatorname{ker} \mu^{p} \subset P_{r c p, c h}(\mathscr{V})$. And so, $D_{\infty} \in \operatorname{ker} \mu^{P}$. Because of $\mu^{p}\left(D_{\infty}\right) \leq \mu^{p}\left(D_{n}\right)$ for all $n$, we obtain $\mu^{p}\left(D_{\infty}\right)=0$. Then, $D_{\infty} \in \operatorname{ker} \mu^{p}$. The measure $\mu^{p}$ is called sublinear if it satisfies

(5) $\mu^{p}\left(D_{1}+D_{2}\right) \leq \mu^{p}\left(D_{1}\right)+\mu^{p}\left(D_{2}\right) \quad$ for $\quad$ all $D_{1}, D_{2} \in P_{b d, c h}(\mathscr{V})$.

(6) $\mu^{p}(\lambda D)=|\lambda| \mu^{p}(D)$ for $\lambda \in \mathbb{R}$.

And $\mu^{p}$ has the maximum property if

(7) $\mu^{p}\left(D_{1}+D_{2}\right)=\max \left\{\mu^{p}\left(D_{1}\right), \mu^{p}\left(D_{2}\right)\right\}$.

Finally, $\mu^{p}$ is said to be full if

(8) $\operatorname{ker} \mu^{p}=P_{r c p, c h}(\mathscr{V})$.

Definition 7 (see [28]). A mapping $\chi:[0, \infty) \longrightarrow[0, \infty)$ is called a $\mathscr{D}$-function if it is monotone nondecreasing and upper semicontinuous with $\chi(0)=0$.

Definition 8 (see [28]). A nondecreasing mapping $\Gamma: \mathscr{V} \longrightarrow \mathscr{V}$ is said to be partially nonlinear $\mathscr{D}$-set-Lipschitz if there is a $\mathscr{D}$-function $\chi$ such that

$$
\mu^{p}(\Gamma \mathscr{C}) \leq \chi\left(\mu^{p}(\mathscr{C})\right),
$$

for any bounded chain $\mathscr{C}$ in $\mathscr{V}$. If $\chi(s)<s$ for $s>0$, then $\Gamma$ is called a partially nonlinear $\mathscr{D}$-set-contraction.

Lemma 2 (see [28]). If $\chi$ is a D-function with $\chi(s)<s$ for $s>0$, then $\lim _{n \longrightarrow \infty} \chi^{n}(s)=0$ for all $s \in[0, \infty)$ and viceversa.

Definition 9 (see [27]). Let $\mathscr{M}, \mathcal{N}$ be nonempty subsets of $\mathscr{X}$. A map $\Gamma: \mathscr{M} \cup \mathscr{N} \longrightarrow \mathscr{M} \cup \mathcal{N}$ is a partially cyclic contraction map if it satisfies

(1) $\Gamma(\mathscr{M}) \subseteq \mathscr{N}$ and $\Gamma(\mathscr{N}) \subseteq \mathscr{M}$

(2) $d(\Gamma \eta, \Gamma \xi) \leq k d(\eta, \xi)+(1-k) d(\mathscr{M}, \mathcal{N})$, for some $k \in(0,1)$ and for $\eta \in \mathscr{M}, \xi \in \mathcal{N}$, with $\eta$ and $\xi$ are comparable

From (2), one can easily verify that $d(\Gamma \eta, \Gamma \xi) \leq d(\eta, \xi)$ holds.

Lemma 3 (see [18]). Let $\mathscr{M}, \mathcal{N}$ be nonempty subsets of $\mathscr{X}$. Suppose $\Gamma: \mathscr{M} \cup \mathscr{N} \longrightarrow \mathscr{M} \cup \mathscr{N}$ is a cyclic contraction map. Then, for any $\eta_{0}$ in $\mathscr{M} \cup \mathcal{N}$, we have $d\left(\eta_{n}, \Gamma \eta_{n}\right) \longrightarrow$ $d(\mathscr{M}, \mathcal{N})$, where $\eta_{n+1}=\Gamma \eta_{n}, n=0,1,2,3, \ldots$

First, we show the result for a single operator. 
Theorem 5. Let $\mathscr{M}, \mathcal{N}$ be two nonempty closed and partially bounded subsets of $(\mathscr{V}, \prec,\|\cdot\|)$. Let $\Gamma: \mathscr{M} \cup \mathcal{N} \longrightarrow \mathscr{M} \cup \mathcal{N}$ be nondecreasing, partially cyclic contraction, and partially nonlinear $\mathscr{D}$-set contraction in $\mathscr{M} \cup \mathcal{N}$. If there exists an element $\eta_{0} \in \mathscr{M}$ such that $\eta_{0} \prec \Gamma \eta_{0}$, then $\Gamma$ has a best proximity point.

Proof. Since $\Gamma$ is cyclic, for $\eta_{0} \in \mathscr{M}$, then $\Gamma \eta_{0} \in \mathcal{N}$. Define $\eta_{1}=\Gamma \eta_{0}$. Now, again $\Gamma \eta_{1} \in \mathscr{M}$, and define $\eta_{2}=\Gamma \eta_{1}$. In the same way, we construct $\eta_{n+1}=\Gamma \eta_{n}$ such that $\left\{\eta_{2 n}\right\} \subseteq \mathscr{M}$ and $\left\{\eta_{2 n+1}\right\} \subseteq \mathcal{N}$, for $n=0,1,2, \ldots$. Since $\eta_{0}<\Gamma \eta_{0}$ and $\Gamma$ is nondecreasing, it implies that $\eta_{1} \prec \eta_{2}$. Again using $\Gamma$ is nondecreasing, we obtain $\eta_{0} \prec \eta_{1} \prec \bar{\eta}_{2} \prec \cdots$. Denote

$$
D_{2 n}=\left\{\eta_{2 n}, \eta_{2 n+2}, \eta_{2 n+4}, \ldots\right\} \text {, }
$$

for $n=0,1,2, \ldots$ Here, each $D_{2 n}$ is a bounded chain in $\mathscr{M}$, and $D_{2 n}=\Gamma^{2}\left(D_{2 n-2}\right), n=1,2, \ldots$. From the construction of $\left\{D_{2 n}\right\}$, we obtain

$$
D_{0} \supseteq D_{2} \supseteq D_{4} \supseteq \cdots
$$

Consequently,

$$
\bar{D}_{0} \supseteq \bar{D}_{2} \supseteq \bar{D}_{4} \supseteq \cdots
$$

Now,

$$
\begin{aligned}
\mu^{p}\left(D_{2 n}\right) & =\mu^{p}\left(\Gamma^{2}\left(D_{2 n-2}\right)\right) \\
& \leq \chi\left(\mu^{p}\left(\Gamma D_{2 n-2}\right)\right) \\
& \leq \chi^{2}\left(\mu^{p}\left(D_{2 n-2}\right)\right) \\
& \leq \chi^{4}\left(\mu^{p}\left(D_{2 n-4}\right)\right) \\
& \vdots \\
& \leq \chi^{2 n}\left(\mu^{p}\left(D_{0}\right)\right) .
\end{aligned}
$$

Letting limit superior as $n \longrightarrow \infty$ and using Lemma 2, we obtain

$$
\begin{aligned}
\mu^{p}\left(\bar{D}_{2 n}\right) & =\lim _{n \longrightarrow \infty} \mu^{p}\left(D_{2 n}\right) \leq \lim _{n \longrightarrow \infty} \sup \chi^{2 n}\left(\mu^{p}\left(D_{0}\right)\right) \\
& =\lim _{n \longrightarrow \infty} \chi^{2 n}\left(\mu^{p}\left(D_{0}\right)\right)=0 .
\end{aligned}
$$

Therefore, by condition (4) of $\mu^{p}$, we get

$$
\bar{D}_{\infty}=\cap_{n=1}^{\infty} D_{2 n} \neq \varnothing, \quad D_{\infty} \in P_{r c p, c h} .
$$

Since $\lim _{n \rightarrow \infty} \mu^{p}\left(D_{2 n}\right)=0$, then for $\varepsilon>0$, there exists $n_{0} \in \mathbb{N}$ such that $\mu^{p}\left(D_{2 n}\right)<\varepsilon$, for $n \geq n_{0}$. This implies that $\bar{D}_{2 n_{0}}$ and $\bar{D}_{0}$ are compact chains in $\mathscr{M}$. Hence, $\left\{\eta_{2 n}\right\}$ has a convergent subsequence, that is, $\eta_{2 n_{k}} \longrightarrow \eta \in \mathscr{M}$. Now,

$$
d(\mathscr{M}, \mathcal{N}) \leq\left\|\eta-\eta_{2 n_{k}-1}\right\| \leq\left\|\eta-\eta_{2 n_{k}}\right\|+\left\|\eta_{2 n_{k}}-\eta_{2 n_{k}-1}\right\| .
$$

As $k \longrightarrow \infty$ and by Lemma 3 , we obtain

$$
d(\mathscr{M}, \mathcal{N}) \leq \lim _{k \longrightarrow \infty}\left\|\eta-\eta_{2 n_{k}-1}\right\| \leq d(\mathscr{M}, \mathcal{N}) .
$$

Therefore, $\lim _{k \rightarrow \infty}\left\|\eta-\eta_{2 n_{k}-1}\right\|=d(\mathscr{M}, \mathcal{N})$. Since $\Gamma$ is a partially cyclic contraction, we have

$$
d(\mathscr{M}, \mathcal{N}) \leq\left\|\eta_{2 n_{k}}-\Gamma \eta\right\| \leq\left\|\eta_{2 n_{k}-1}-\eta\right\| .
$$

As $k \longrightarrow \infty$, we get

$$
d(\mathscr{M}, \mathcal{N}) \leq \lim _{k \longrightarrow \infty}\left\|\eta_{2 n_{k}}-\Gamma \eta\right\| \leq d(\mathscr{M}, \mathcal{N}),
$$

which implies that $\|\eta-\Gamma \eta\|=d(\mathscr{M}, \mathcal{N})$.

Using the above theorem, here, we provide the result on the best proximity point for the sum of two operators.

Theorem 6. Let $\mathscr{M}, \mathcal{N}$ be a nonempty, closed, and partially bounded subset of $(\mathscr{V}, \prec,\|\cdot\|)$. Let $\Gamma_{1}, \Gamma_{2}: \mathscr{M} \cup \mathcal{N} \longrightarrow \mathscr{M} \cup \mathcal{N}$ be nondecreasing cyclic mappings which satisfy

(1) $\eta \in \mathscr{M} \Longrightarrow \Gamma_{1} \eta+\Gamma_{2} \eta \in \mathcal{N}$ and $\eta \in \mathscr{N} \Longrightarrow \Gamma_{1} \eta+\Gamma_{2} \eta \in \mathscr{M}$

(2) $\Gamma_{1}$ is partially completely continuous, and

$$
\left\|\Gamma_{1} \eta-\Gamma_{1} \xi\right\| \leq \frac{k}{2}\|\eta-\xi\|+\frac{1}{2} d(\mathscr{M}, \mathcal{N}),
$$

where $k \in(0,(1 / 2))$ and $\eta \in \mathscr{M}, \xi \in \mathcal{N}$ with $\eta$ and $\xi$ are comparable.

(3) $\Gamma_{2}$ is a nonlinear $\mathscr{D}$-set contraction on $\mathscr{M}$, and

$$
\left\|\Gamma_{2} \eta-\Gamma_{2} \xi\right\| \leq \frac{k}{2}\|\eta-\xi\|
$$

where $k \in(0,(1 / 2))$ and $\eta \in \mathscr{M}, \xi \in \mathcal{N}$ with $\eta$ and $\xi$ are comparable.

(4) There exists an element $\eta_{0} \in \mathscr{M}$ such that $\eta_{0} \preceq \Gamma_{1} \eta_{0}+\Gamma_{2} \eta_{0}$.

Then, there exists $\eta^{*} \in \mathscr{M}$ such that $\left\|\eta^{*}-\left(\Gamma_{1}+\Gamma_{2}\right) \eta^{*}\right\|=d(\mathscr{M}, \mathcal{N})$.

Proof. Define $\quad \Gamma_{1}+\Gamma_{2}: \mathscr{M} \cup \mathcal{N} \longrightarrow \mathscr{M} \cup \mathcal{N} \quad$ by $\left(\Gamma_{1}+\Gamma_{2}\right)(\eta)=\Gamma_{1}(\eta)+\Gamma_{2}(\eta)$. Clearly, because of $(1), \Gamma_{1}+\Gamma_{2}$ is a cyclic mapping. And one can easily prove $\Gamma_{1}+\Gamma_{2}$ is nondecreasing on $\mathscr{M} \cup \mathcal{N}$. Since $k<(1 / 2)$ implies $(1 / 2)<1-k$, now, we show that $\Gamma_{1}+\Gamma_{2}$ is a partial contraction. For $\eta \in \mathscr{M}$ and $\xi \in \mathscr{N}$ with $\eta$ and $\xi$ are comparable, we have

$$
\begin{aligned}
\left\|\left(\Gamma_{1}+\Gamma_{2}\right)(\eta)-\left(\Gamma_{1}+\Gamma_{2}\right)(\xi)\right\| & =\left\|\Gamma_{1} \eta+\Gamma_{2} \eta-\Gamma_{1} \xi-\Gamma_{2} \xi\right\| \\
& \leq\left\|\Gamma_{1} \eta-\Gamma_{1} \xi\right\|+\left\|\Gamma_{2} \eta-\Gamma_{2} \xi\right\| \\
& \leq \frac{k\|\eta-\xi\|}{2}+\frac{d(\mathscr{M}, \mathcal{N})}{2}+\frac{k\|\eta-\xi\|}{2} \\
& \leq k\|\eta-\xi\|+(1-k) d(\mathscr{M}, \mathcal{N}),
\end{aligned}
$$

which show that $\Gamma_{1}+\Gamma_{2}$ is a partial contraction. Next, we prove that $\Gamma_{1}+\Gamma_{2}$ is a partially nonlinear $\mathscr{D}$-set contraction. 
Let $\mathscr{C}$ be a bounded chain in $\mathscr{M}$. Therefore, $\left(\Gamma_{1}+\Gamma_{2}\right)(\mathscr{C}) \subseteq \Gamma_{1}(\mathscr{C})+\Gamma_{2}(\mathscr{C})$. By conditions sublinearity and full of $\mu^{p}$, we get

$$
\begin{aligned}
\mu^{p}\left(\left(\Gamma_{1}+\Gamma_{2}\right)(\mathscr{C})\right) & \leq \mu^{p}\left(\Gamma_{1}(\mathscr{C})+\Gamma_{2}(\mathscr{C})\right) \\
& \leq \mu^{p}\left(\Gamma_{1}(\mathscr{C})\right)+\mu^{p}\left(\Gamma_{2}(\mathscr{C})\right) \\
& \leq \chi\left(\mu^{p}(\mathscr{C})\right) .
\end{aligned}
$$

Therefore, the mapping $\Gamma_{1}+\Gamma_{2}$ follows Theorem 5 , and there exists $\eta^{*} \in \mathscr{M}$ such that $\| \eta^{*}-\left(\Gamma_{1}+\Gamma_{2}\right)$ $\left(\eta^{*}\right) \|=d(\mathscr{M}, \mathcal{N})$.

\section{Conclusions}

In the nonlinear functional analysis, many mathematical problems can be solved by the existence result of fixed points. The fixed point theorems provide sufficient conditions to ensure the fixed point equation $\Gamma x=x$, where $\Gamma$ is the self-mapping, has a solution. In case of nonlinear problems, it is written as $\Gamma_{1} x+\Gamma_{2} x=x$, where $\Gamma_{1}, \Gamma_{2}$ are selfmappings, and then the fixed point theorems for the sum of two mappings help to obtain the solution for such an equation. Suppose the mappings $\Gamma_{1}, \Gamma_{2}$ are non-self-cases, then the fixed point equation $\Gamma_{1} x+\Gamma_{2} x=x$ does not possess a solution. In the literature, there are many research papers which deal with the existence of the best proximity point for the equation of the form $\Gamma x=x$, where $\Gamma$ is the non-selfmapping. However, there is no single research work which gives the existence result of the best proximity point for the sum of mappings. So, we want to obtain an approximate solution via finding the best proximity point for such an equation in some sense. So, in this research article, we study the existence of the best proximity point for the sum of two non-self-mappings using best proximity point theorems for a single operator. Moreover, using the notion accretive operators, we prove an existence result of the fixed point for the sum of cyclic and noncyclic operators. Also, we study some notions of the ordered normed linear space, and we provide sufficient conditions for the existence of the best proximity point.

\section{Data Availability}

No data were used to support this study.

\section{Conflicts of Interest}

The authors declare that they have no conflicts of interest.

\section{Acknowledgments}

This work was partially funded by Basque Government through Grant IT1207-19.

\section{References}

[1] B. C. Dhage and D. Otrocol, "Dhage iteration method for approximating solutions of nonlinear differential equations with maxima," Fixed Point Theory, vol. 19, no. 2, pp. 545-556, 2018.
[2] J. Banas and A. B. Amar, "Measures of noncompactness in locally convex spaces and fixed point theory for the sum of two operators on unbounded convex sets," Commentationes Mathematicae Universitatis Carolinae, vol. 54, no. 1, pp. 2140, 2013.

[3] B. C. Dhage, "Remarks on two fixed-point theorems involving the sum and the product of two operators," Computers \& Mathematics with Applications, vol. 46, no. 12, pp. 1779-1785, 2003.

[4] B. C. Dhage, "Local fixed point theory for the sum of two operators in Banach spaces," Fixed Point Theory, vol. 4, pp. 49-60, 2003.

[5] D. O'Regan, "Fixed-point theory for the sum of two operators," Applied Mathematics Letters, vol. 9, pp. 1-8, 1996.

[6] D. O'Regan and M. A. Taoudi, "Fixed point theorems for the sum of two weakly sequentially continuous mappings," Nonlinear Analysis, vol. 73, pp. 283-289, 2010.

[7] P. Vijayaraju, "Fixed point theorems for a sum of two mappings in locally convex spaces," International Journal of Mathematics and Mathematical Sciences, vol. 17, no. 4, pp. 681-686, 1994.

[8] R. P. Agarwal, D. O'Regan, and M. A. Taoudi, "BrowderKrasnoselskii-type fixed point theorems in Banach spaces," Fixed Point Theory and Applications, vol. 2010, Article ID 243716, 20 pages, 2010.

[9] A. Ben Amar and J. Garcia-Falset, "Fixed point theorems for 1-set weakly contractive and pseudocontractive operators on an unbounded domain," Portugaliae Mathematica, vol. 68, no. 2, pp. 125-147, 2011.

[10] A. Arunchai and S. Plubtieng, "On the Krasnoselskii-type fixed point theorems for the sum of continuous and asymptotically nonexpansive mappings in Banach spaces," Journal of Inequalities and Applications, vol. 2011, no. 1, p. 11, 2011.

[11] F. Wang, "Fixed-point theorems for the sum of two operators under $\omega$-condensing," Fixed Point Theory and Applications, vol. 2013, no. 1, p. 13, 2013.

[12] A. B. Amar, D. O'Regan, and A. Touati, "Fixed point theorems for the sum of $(w s)$-compact and asymptotically $\Phi$-nonexpansive mappings," Journal of Fixed Point Theory and Applications, vol. 18, no. 4, pp. 771-784, 2016.

[13] A. C. M. Ran and M. C. B. Reurings, "A fixed point theorem in partially ordered sets and some applications to matrix equations," Proceedings of the American Mathematical Society, vol. 132, no. 05, pp. 1435-1444, 2004.

[14] J. J. Nieto and R. Rodríguez-López, "Contractive mapping theorems in partially ordered sets and applications to ordinary differential equations," Order, vol. 22, no. 3, pp. 223-239, 2005.

[15] A. Petruşel and I. A. Rus, "Fixed point theorems in ordered L-spaces," Proceedings of the American Mathematical Society, vol. 134, pp. 411-418, 2006.

[16] S. S. Basha, "Best proximity point theorems," Journal of Approximation Theory, vol. 163, no. 11, pp. 1772-1781, 2011.

[17] S. S. Basha, "Best proximity point theorems for contractive mappings," Journal of Fixed Point Theory and Applications, vol. 20, p. 16, 2018.

[18] A. A. Eldred and P. Veeramani, "Existence and convergence of best proximity points," Journal of Mathematical Analysis and Applications, vol. 323, no. 2, pp. 1001-1006, 2006.

[19] M. A. Al-Thagafi and N. Shahzad, "Convergence and existence results for best proximity points," Nonlinear Analysis: Theory, Methods \& Applications, vol. 70, no. 10, pp. 36653671, 2009. 
[20] V. Sankar Raj, "A best proximity point theorem for weakly contractive non-self-mappings," Nonlinear Analysis: Theory, Methods \& Applications, vol. 74, no. 14, pp. 4804-4808, 2011.

[21] S. Sadiq Basha and P. Veeramani, "Best proximity pair theorems for multifunctions with open fibres," Journal of Approximation Theory, vol. 103, no. 1, pp. 119-129, 2000.

[22] W. K. Kim and K. H. Lee, "Existence of best proximity pairs and equilibrium pairs," Journal of Mathematical Analysis and Applications, vol. 316, no. 2, pp. 433-446, 2006.

[23] C. Mongkolkeha and P. Kumam, "Best proximity point theorems for generalized cyclic contractions in ordered metric spaces," Journal of Optimization Theory and Applications, vol. 155, no. 1, pp. 215-226, 2012.

[24] A. Abkar and M. Gabeleh, "Best proximity points for cyclic mappings in ordered metric spaces," Journal of Optimization Theory and Applications, vol. 150, no. 1, pp. 188-193, 2011.

[25] V. Pragadeeswarar and M. Marudai, "Best proximity points: approximation and optimization in partially ordered metric spaces," Optimization Letters, vol. 7, no. 8, pp. 1883-1892, 2013.

[26] W. Takahashi, Nonlinear Functional Analysis: Fixed Point Theory and Its Applications, Yokohama Publishers, Yokohama, Japan, 2000.

[27] W. A. Kirk, P. S. Srinivasan, and P. Veeramani, "Fixed points for mappings satisfying cyclical contractive conditions," Fixed Point Theory, vol. 4, pp. 79-89, 2003.

[28] B. C. Dhage, "Nonlinear D-set contraction mappings in partially ordered normed linear spaces and applications to functional hybrid integral equations," Malaya Journal of Matematik, vol. 3, no. 1, pp. 62-85, 2015. 